Artigo Original

Original Article

Amanda Rodrigues Scheffer ${ }^{1}$ (1) Maria Carolina Ferreira ${ }^{1}$ (C) Maria Fernanda Capoani Garcia Mondelli² (C)

Descritores

Adulto

Audição

Estudos Transversais

Perda Auditiva

Zumbido

Keywords

Adult

Hearing

Cross-Sectional Studies

Hearing Loss

Tinnitus

Endereço para correspondência: Maria Carolina Ferreira

Alameda Doutor Octávio Pinheiro Brisolla 9-75, Vila Universitária - CEP: 17012-901, Bauru (SP), Brasil.

E-mail: mariaferrreira@usp.br

Recebido em: Janeiro 28, 2020

\section{Aplicabilidade do Tinnitus and Hearing Survey (THS) na diferenciação de queixas auditivas}

\author{
The applicability of the Tinnitus and Hearing \\ Survey (THS) in the differentiation of tinnitus
} and hearing complaints

\section{RESUMO}

Objetivo: verificar a aplicabilidade do THS em mensurar e diferenciar as queixas de zumbido, perda auditiva e tolerância ao som. Método: Foi aplicado o questionário THS, composto por 4 questões sobre zumbido, 4 sobre perda auditiva e 1 sobre tolerância ao som. Previamente, todos os participantes realizaram uma bateria de exames de diagnóstico audiológico e foram divididos em grupos: audição normal bilateral com média até $25 \mathrm{~dB}$, com e sem queixa de zumbido (Grupos 1 e 3); diagnóstico de perda auditiva sensório neural de grau leve a moderado (26dB a $60 \mathrm{~dB}$ ), sem uso prévio de aparelho de amplificação sonora Individual (AASI), com queixa de zumbido crônico ( $\geq 6$ meses) e também indivíduos sem queixa de zumbido (Grupos 2 e 4); idade igual ou superior a 18 anos. Resultados: Foram incluídos no presente estudo 70 indivíduos. Em relação a análise do total entre os grupos pelo teste Kruskal-Wallis foram encontradas diferenças significativas nas seções sobre zumbido e perda auditiva, mas não houve significância da seção sobre tolerância ao som. Em relação às questões sobre zumbido apenas os grupos 3 e 4 pontuaram. Quanto às questões referentes à perda auditiva, os grupos sem perda (grupos 1 e 3) foram os que menos pontuaram. Em relação a questão do THS sobre tolerância ao som, o grupo 3 foi o que mais pontuou. Conclusão: o questionário THS se mostrou como uma possível ferramenta para auxiliar na compreensão e diferenciação das queixas auditivas.

\begin{abstract}
Purpose: to verify the applicability of Tinnitus and Hearing Survey (THS) to measure and differentiate complaints of tinnitus, hearing loss and sound tolerance. Methods: THS was performed, composed by 4 questions about tinnitus, 4 about hearing loss and one about sound tolerance. Previously, all participants performed a battery of audiological diagnostic tests and then divided into 4 groups: Bilateral normal audiometry with mean up to $25 \mathrm{~dB}$, with and without tinnitus complaint (Groups 1 and 3); diagnosis of mild to moderate neural sensory hearing loss ( $26 \mathrm{~dB}$ to $60 \mathrm{~dB})$, no previous use of individual hearing aids, complaining of chronic tinnitus ( $\geq 6$ months) and, individuals without tinnitus complaints (Groups 2 and 4); and age $\geq 18$ years old. Results: Seventy subjects were included in this study. Regarding the analysis of the total between the groups by the Kruskal-Wallis test, significant differences were found in sections about tinnitus and hearing Loss, but there was no significance in section of sound tolerance. Regarding the questions in Section A of the THS, only groups 3 and 4 scored. Regarding the questions related to Section B about hearing loss, the groups without hearing loss (groups 1 and 3) scored the lowest. In relation to the question of THS of Section C, group 3 scored the highest. Conclusion: The THS questionnaire proved to be a useful, quick and simple tool to assist the audiologist in the understanding and differentiation of the audiologic complaints.
\end{abstract}

Trabalho realizado na Faculdade de Odontologia de Bauru, Universidade de São-FOB/USP, Bauru (SP), Brasil. ${ }^{1}$ Programa de Pós-graduação, Faculdade de Odontologia de Bauru - FOB, Universidade de São Paulo - USP - Bauru (SP), Brasil.

${ }^{2}$ Departamento de Fonoaudiologia, Faculdade de Odontologia de Bauru - FOB, Universidade de São Paulo USP - Bauru (SP), Brasil.

Fonte de financiamento: nada a declarar.

Conflito de interesses: nada a declarar 


\section{INTRODUÇÃO}

A perda auditiva pode causar comprometimentos psicossociais e consequências negativas para o indivíduo. A dificuldade causada por ela no decorrer dos anos a torna um fator de afastamento social e das atividades ocupacionais, gerando mudanças nas relações interpessoais dessa população $0^{(1,2)}$.

Há diversos fatores e sintomas relacionados à perda auditiva, como hipertensão arterial, diabetes mellitus e problemas vestibulares. Em relação às queixas auditivas tem sido relatado, com frequência, o zumbido - uma percepção auditiva notada apenas pelo indivíduo acometido podendo causar problemas de concentração, dificuldade para dormir, irritação, afastamento social e abalo emocional ${ }^{(3-6)}$.

A relação entre perda auditiva e zumbido já foi muito citada ${ }^{(7,8)}$. O zumbido crônico é frequentemente acompanhado por uma deficiência auditiva e geralmente, a perda auditiva sensório neural de grau moderado está presente em associação a esse sintoma, afetando amplamente a comunicação e a vida desses indivíduos ${ }^{(9)}$.

Ainda associado às queixas de zumbido, existe a reduzida tolerância ao som. Problemas de tolerância ao som são mais prováveis de ocorrer em indivíduos afetados pelo zumbido, e o uso de uma conceituação biopsicossocial do zumbido e outras condições de medicina comportamental poderiam ser úteis para entender e tratar tais problemas ${ }^{(10)}$.

Com base na literatura sobre zumbido, é possível estimar a prevalência da baixa tolerância sonora na população geral. No estudo realizado pela Emory Tinnitus and Hyperacusis Center em Atlanta, $60 \%$ dos pacientes examinados com zumbido têm baixa tolerância sonora, contudo, este problema apenas atraiu atenção recentemente ${ }^{(11,12)}$.

O grau de incômodo causado pelo zumbido está relacionado ao incômodo causado pela perda auditiva, o que pode justificar a errônea atribuição das dificuldades auditivas ao zumbido por parte de pacientes que apresentam o sintoma, o que torna essencial a separação dos problemas causados pelo zumbido dos problemas causados pela perda de audição, para que o direcionamento do tratamento seja mais específico proporcionando uma maior eficácia da intervenção ${ }^{(13,14)}$.

Os questionários atuais de autorrelato a respeito do zumbido, predizem que as queixas auditivas estão relacionadas ao próprio sintoma, atribuindo muitas vezes perguntas sobre o impacto do zumbido nas atividades de vida diárias, trabalho e questões sociais, dificultando ainda mais a determinação de qual queixa auditiva realmente incomoda o paciente, sendo que por vezes, o indivíduo atribui a dificuldade de ouvir apenas ao sintoma. Existe uma população que apresenta apenas o zumbido, sem a perda auditiva, para estes, a intervenção se difere, ressaltando a importância da diferenciação das queixas, visto que, algumas intervenções para o zumbido, não levam em consideração a perda auditiva. Desta forma, quando esclarecidas e definidas as queixas auditivas, pode-se estudar estratégias de intervenção mais eficientes e que realmente atendam às necessidades dos pacientes ${ }^{(13)}$.

Ainda hoje, existe uma grande dificuldade de mensurar e diferenciar os sintomas do zumbido das queixas auditivas, tanto pelo paciente, que muitas vezes não consegue relatar o que o incomoda mais, quanto pelo terapeuta, uma vez que não há consenso pleno na literatura quanto a um instrumento de avaliação.
Desta forma, o Tinnitus and Hearing Survey (THS) foi desenvolvido, com a proposta de identificar o quanto da queixa do paciente está relacionada aos problemas auditivos e o quanto está relacionada diretamente ao zumbido, bem como, este questionário pode facilitar o esclarecimentos dos direcionamentos para identificar problemas de tolerância sonora e identificar o melhor tratamento para cada paciente ${ }^{(13)}$. OTHS é dividido em três seções, A, B, C, sendo que para cada uma foram atribuídas questões relacionadas especificamente ao zumbido, à perda auditiva e à tolerância sonora, possibilitando uma rápida avaliação dos efeitos da perda auditiva separados dos efeitos ocasionados pelo zumbido ${ }^{(15,16)}$. Desta forma, o objetivo deste trabalho foi verificar a aplicabilidade do THS em mensurar e diferenciar as queixas de zumbido, perda auditiva e tolerância ao som.

\section{MÉTODO}

Realizou-se um estudo observacional, do tipo transversal, na Clínica de Fonoaudiologia da Faculdade de Odontologia de Bauru (FOB), da Universidade de São Paulo (USP), sob aprovação do Comitê de Ética em Pesquisas (CEP) CAAE: 59804216.1.0000.5417.

Todos os participantes selecionados assinaram o Termo de Consentimento Livre e Esclarecido (TCLE) e passaram por uma avaliação prévia, na qual foram realizados os seguintes exames: Audiometria Tonal Liminar, Logoaudiometria e Medidas de Imitância Acústica.

No total foram selecionadas 70 pessoas de forma aleatória conforme os critérios de elegibilidade, com e sem queixa de zumbido e de ambos os sexos para participação voluntária no presente estudo. A amostra foi distribuída em quatro grupos: 20 indivíduos sem perda auditiva e sem zumbido (Grupo 1); 14 indivíduos com perda auditiva e sem zumbido (Grupo 2); 14 indivíduos sem perda auditiva e com zumbido (Grupo 3); 22 indivíduos com perda auditiva e com zumbido (Grupo 4).

\section{Critérios de Inclusão:}

- Audiometria normal bilateral com média estabelecida pela Organização Internacional de Normalização (ISO) de 500, 1000, 2000, e $4000 \mathrm{~Hz}$ até $25 \mathrm{~dB}$ e sem queixa de zumbido (Grupo 1);

- Diagnóstico de perda auditiva sensório neural de grau leve a moderado, com média ISO de $26 \mathrm{~dB}$ a $60 \mathrm{~dB}$, sem uso prévio de aparelho de amplificação sonora Individual (AASI) e sem queixa de zumbido (Grupo 2);

- Audiometria normal bilateral, com média ISO até $25 \mathrm{~dB}$ e com queixa de zumbido contínuo por um período mínimo de 6 meses (Grupo 3);

- Diagnóstico de perda auditiva sensório neural de grau leve a moderado, com média ISO de $26 \mathrm{~dB}$ a $60 \mathrm{~dB}$, sem uso prévio de AASI e com queixa de zumbido contínuo por um período mínimo de 6 meses (Grupo 4);

- Indivíduos que não apresentassem limitações de comparecimento a clínica e de responder ao questionário;

- Ter idade igual ou superior a 18 anos. 


\section{Critérios de Exclusão:}

- Perda auditiva sensório neural de grau severo ou profundo com média ISO igual ou superior à $61 \mathrm{~dB}$;

- Uso prévio de AASI;

- Apresentar zumbido esporádico;

- Realização prévia de algum tipo de intervenção ao zumbido.

Para as avaliações iniciais referentes ao diagnóstico audiológico, foi realizado a Audiometria Tonal Liminar com estímulo Warble Tone e aplicação do método descendente, nas frequências de $250 \mathrm{~Hz}$, $500 \mathrm{~Hz}, 1000 \mathrm{~Hz}, 2000 \mathrm{~Hz}, 3000 \mathrm{~Hz}, 4000 \mathrm{~Hz}, 6000 \mathrm{~Hz}$ e $8000 \mathrm{~Hz}$ para classificar a acuidade auditiva do paciente, a partir das médias das frequências de $500 \mathrm{~Hz}, 1000 \mathrm{~Hz}, 2000 \mathrm{~Hz}$ e $4000 \mathrm{~Hz}$. Considerou-se perda auditiva todo resultado acima da média de 26 dBNA.

A logoaudiometria foi realizada com o objetivo de medir a capacidade de o paciente detectar e reconhecer a fala, por meio da análise do Índice Percentual de Reconhecimento de Fala (IPRF), onde acrescemos $40 \mathrm{~dB}$ a média de $500 \mathrm{~Hz}, 1000 \mathrm{~Hz}$, $2000 \mathrm{~Hz}$ e $4000 \mathrm{~Hz}$ para cada orelha do paciente e solicitamos que ele repetisse 25 palavras monossilábicas.

As medidas de Imitância acústica foram realizadas, a fim de descartar possíveis alterações de orelha externa/média, bem como alterações retrococleares nos integrantes da pesquisa ${ }^{(17)}$.

Posteriormente as avaliações, um entrevistador, conhecedor das perguntas que compunham o THS, aplicou o questionário adaptado em todos os participantes da pesquisa ${ }^{(6)}$.

O THS é dividido em três seções, sendo a primeira a seção A, a qual corresponde a quatro questões apenas sobre zumbido (dificuldade de concentração, relaxamento e também dificuldades relacionadas ao sono), que não estão relacionadas com a perda auditiva. Seção $\mathrm{B}$, composta por quatro questões relacionadas apenas com a perda auditiva (dificuldade em entender fala no ruído, conversações em grupos e vozes com reduzida intensidade). Seção C, composta por uma questão sobre tolerância ao som, a qual não está relacionada às dificuldades decorrentes do zumbido ou da perda auditiva ${ }^{(18)}$. Desta forma, os pacientes poderiam atribuir as seguintes notas para cada questão: 0 (não, não é um problema), 1 (sim, é um problema pequeno), 2 (sim, é um problema moderado), 3 (sim, é um problema grande) ou 4 (sim, é um problema muito grande) (Anexo 1).

Ao final do teste deve ser somado o total de cada coluna e posteriormente o total geral da seção, sendo a sessão que apresentar maior pontuação, aquela à qual a queixa é mais evidente.

Logo após completar o THS, os pacientes podem compreender de forma mais clara que os problemas listados na seção A do questionário, estão relacionados ao gerenciamento do zumbido. Os problemas reportados na seção B estão relacionados a intervenções específicas para perda auditiva e as dificuldades apresentadas na aplicação da seção $\mathrm{C}$ do questionário, estão relacionadas aos problemas de intolerância sonora ${ }^{(19)}$.

Os dados foram registrados no Microsoft Office Excel, a partir da distribuição dos pacientes entre grupos, tabulados e descritos de acordo com a análise estatística descritiva (média e desvio padrão) e o teste de Kruskal-Wallis foi utilizado para as análises inferenciais entre os 4 grupos e em seguida foi aplicado o teste de comparações múltiplas de Pairwise para especificar qual a significância encontrada.

\section{RESULTADOS}

A composição dos quatro grupos ficou definida como segue: grupo 1 composto por 13 mulheres (65\%) e sete homens (35\%) com média de idade para as mulheres de $46,2 \pm 14,8$ e para os homens de $28,6 \pm 8,6$, sem perda auditiva e sem zumbido; grupo 2 composto por oito mulheres $(57,1 \%)$ e seis homens $(42,9 \%)$, com média de idade de $62,2 \pm 5,6$ e homens $66,6 \pm 14,0$ e com perda auditiva e sem zumbido, grupo 3 composto por seis mulheres (42,9\%) e oito homens $(57,1 \%)$ com média de idade de $43,3 \pm 19,0$ para as mulheres e de $46,1 \pm 14,5$ para os homens; sem perda auditiva e com zumbido e o grupo 4 composto por nove mulheres $(40,9 \%)$ e 13 homens $(59,1 \%)$ com média de idade de $52,2 \pm 16,5$ entre as mulheres e de $62,3 \pm 9,5$ entre os homens; com perda auditiva e com zumbido. A média geral da amostra foi de 50,8 15,6 anos para as mulheres e de 52,4 $\pm 18,1$ anos para os homens, destacando a homogeneidade do grupo.

A Tabela 1 apresenta a descrição da amostra.

Em relação a análise do total entre os grupos pelo teste Kruskal-Wallis foram encontradas diferenças significativas $(\mathrm{p}<0,05)$ nas seções A e B (Zumbido e Perda Auditiva), mas não houve significância da seção $C$ sobre tolerância ao som $(\mathrm{p}=0,476)$. As Figuras 1-3 apresentam as médias dos totais por grupo segundo a análise descritiva dos resultados.

\section{TOTAL DO ZUMBIDO}

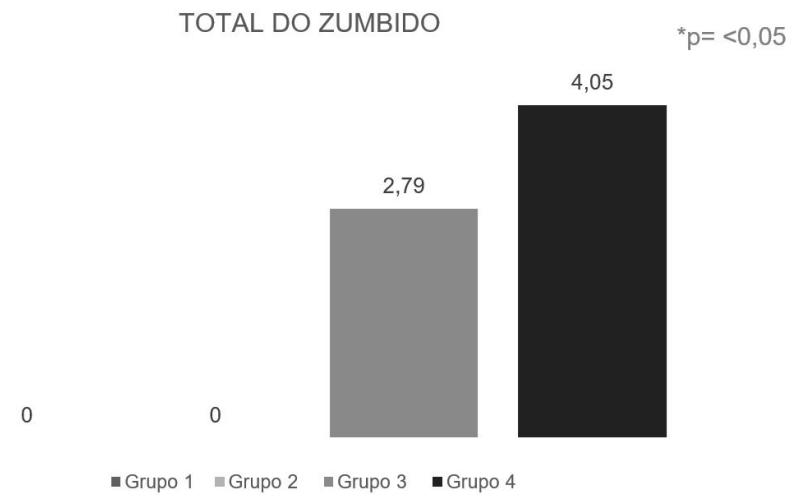

Legenda: Grupo 1= sem zumbido e sem perda auditiva; Grupo 2= com perda auditiva e sem zumbido; Grupo 3= sem perda auditiva e com zumbido; Grupo 4=com perda auditiva e com zumbido; Kruskal-Wallis $(p<0,05)^{\star}$.

Figura 1. Gráfico representativo da média das respostas às questões sobre zumbido do THS entre os 4 grupos.

Tabela 1. Análise descritiva do sexo e perfil das idades da amostra por grupo.

\begin{tabular}{|c|c|c|c|c|c|c|c|c|}
\hline & \multicolumn{2}{|c|}{ Grupo 1} & \multicolumn{2}{|c|}{ Grupo 2} & \multicolumn{2}{|c|}{ Grupo 3} & \multicolumn{2}{|c|}{ Grupo 4} \\
\hline & Mulheres & Homens & Mulheres & Homens & Mulheres & Homens & Mulheres & Homens \\
\hline Amostra (\%) & $13(65 \%)$ & 7 (35\%) & $8(57,1 \%)$ & $6(42,9 \%)$ & $6(42,9 \%)$ & $8(57,1 \%)$ & $9(40,9 \%)$ & $13(59,1 \%)$ \\
\hline Idade (dp) & $46,2(14,8)$ & $28,6(8,6)$ & $62,2(5,6)$ & $66,6(14,0)$ & $43,3(19,0)$ & $46,1(14,5)$ & $52,2(16,5)$ & $62,6(9,5)$ \\
\hline
\end{tabular}

Legenda: $\mathrm{dp}=$ desvio padrão. 
Tabela 2. Análise das comparações do total entre grupos na seção zumbido.

\begin{tabular}{|c|c|c|c|}
\hline Grupos & Estatística do teste & Erro Padrão & $p$ \\
\hline Grupo 2 e Grupo 4 & 31,568 & 6,265 & $0,000^{*}$ \\
\hline Grupo 1 e Grupo 4 & 31,568 & 5,662 & $0,000^{*}$ \\
\hline Grupo 2 e Grupo 3 & 25,393 & 6,926 & $0,000^{*}$ \\
\hline Grupo 1 e Grupo 3 & 25,393 & 6,386 & $0,000^{*}$ \\
\hline Grupo 1 e Grupo 2 & 0,000 & 6,386 & 1,000 \\
\hline Grupo 3 e Grupo 4 & 6,175 & 6,265 & 0,324 \\
\hline
\end{tabular}

Legenda: Grupo 1= sem zumbido e sem perda auditiva; Grupo 2= com perda auditiva e sem zumbido; Grupo 3= sem perda auditiva e com zumbido; Grupo 4= com perda auditiva e com zumbido. Comparações por Método Pairwise de grupo (* $\mathrm{p}<0,05)$.

Tabela 3. Análise das comparações do total entre grupos na seção perda auditiva.

\begin{tabular}{cccc}
\hline Grupos & Estatística do teste & Erro Padrão & $\mathrm{p}$ \\
\hline Grupo 1 e Grupo 3 & 1,357 & 7,001 & 0,846 \\
Grupo 1 e Grupo 2 & 20,679 & 7,001 & $0,003^{*}$ \\
Grupo 1 e Grupo 4 & 28,932 & 6,207 & $0,000^{*}$ \\
Grupo 2 e Grupo 3 & $-19,321$ & 7,593 & $0,011^{*}$ \\
Grupo 3 e Grupo 4 & 27,575 & 6,869 & $0,000^{*}$ \\
Grupo 2 e Grupo 4 & 8,253 & 6,869 & 0,230 \\
\hline
\end{tabular}

Legenda: Grupo 1= sem zumbido e sem perda auditiva; Grupo 2= com perda auditiva e sem zumbido; Grupo 3= sem perda auditiva e com zumbido; Grupo 4= com perda auditiva e com zumbido. Comparações por Método Pairwise de grupo $\left({ }^{*} p<0,05\right)$.

TOTAL DA PERDA AUDITIVA

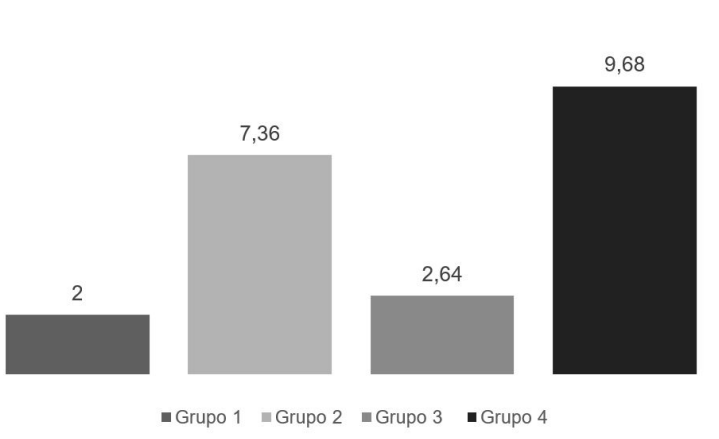

Legenda: Grupo 1= sem zumbido e sem perda auditiva; Grupo 2= com perda auditiva e sem zumbido; Grupo $3=$ sem perda auditiva e com zumbido; Grupo 4= com perda auditiva e com zumbido; Kruskal-Wallis $(p<0.05)^{*}$.

Figura 2. Gráfico representativo da média das respostas às questões sobre perda auditiva do THS entre os 4 grupos.

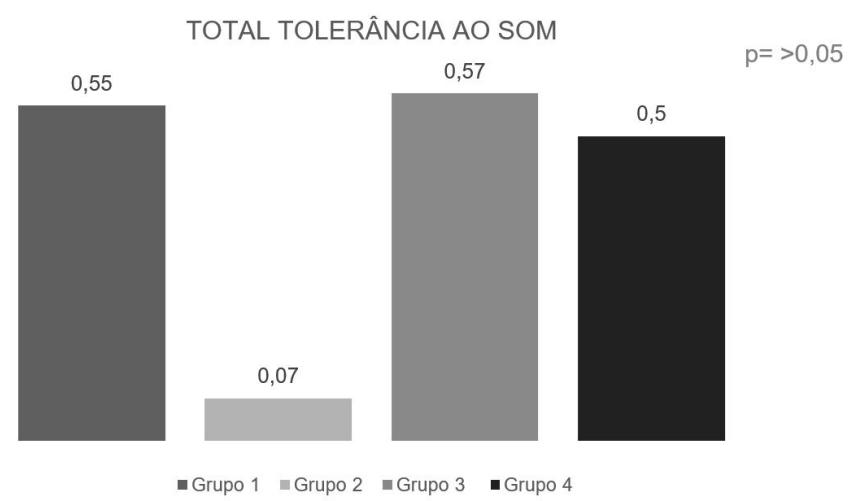

Legenda: Grupo 1= sem zumbido e sem perda auditiva; Grupo 2= com perda auditiva e sem zumbido; Grupo 3= sem perda auditiva e com zumbido; Grupo 4=com perda auditiva e com zumbido; Kruskal-Wallis $(p<0,05)^{\star}$.

Figura 3. Gráfico representativo da média das respostas à questão sobre tolerância ao som do THS entre os 4 grupos.
$\mathrm{Na}$ análise das comparações pelo método Pairwise do total entre grupos, foram encontradas diferenças significativas $(\mathrm{p}<0,05)$ na seção sobre zumbido entre os grupos 2-4; 1-4; 2-3 e 1-3, como mostrado na Tabela 2.

Já na seção Perda Auditiva foram encontradas diferenças significativas $(p<0,05)$ entre os grupos 1-2; 1-4; $2-3$ e 3-4, como mostrado na Tabela 3.

\section{DISCUSSÃO}

O zumbido caracteriza-se por ser um sintoma complexo, referido de diferentes formas e que pode trazer prejuízos à qualidade de vida do indivíduo que o refere. Por vezes a perda auditiva está associada ao sintoma e dificulta o esclarecimento a respeito do que é queixa relacionada ao zumbido e do que é queixa proveniente da perda auditiva. Desta forma, o presente estudo selecionou diferentes grupos de indivíduos para verificar a efetividade e aplicabilidade do THS em mensurar e diferenciar as queixas de zumbido, perda auditiva e tolerância ao som.

Setenta indivíduos foram avaliados por meio da aplicação do THS, sendo a amostra composta por 36 mulheres e 34 homens no total (Tabela 1). Os grupos 3 e 4 que apresentavam o zumbido associado ou não à perda auditiva, apresentaram mais homens do que mulheres com o sintoma, assim como a média de idade desses indivíduos ficou entre 64,1 e 58,4, corroborando com outros estudos ${ }^{(20-23)}$. Por outro lado, os grupos 1 e 2 que incluíram indivíduos que não apresentavam zumbido tiveram uma amostra composta por mais mulheres do que homens. Essa diferença provavelmente foi observada devido a distinta composição dos grupos, pois os indivíduos com e sem zumbido foram alocados em grupos diferentes, inerentemente sendo distintos desde a sua composição. 
Quando realizada a comparação entre os quatro grupos em relação às questões sobre zumbido, os grupos 3 (média=4,05) e 4 (média $=2,79)$ foram os que mais apresentaram queixas, visto que, ambos incluíram indivíduos que apresentavam zumbido (Figura 1).

Averiguou-se que pacientes que não possuem perda auditiva e possuem zumbido, relataram mais incômodo do que aqueles que apresentaram ambos sintomas. Devido à heterogeneidade do sintoma, a interpretação do aborrecimento do zumbido é complexa e depende de muitos fatores inerentes ao indivíduo, devendo-se compreender cada indivíduo dentro de suas especificidades ${ }^{(24)}$.

No entanto, acredita-se que o aborrecimento causado pelo zumbido, bem como a dificuldade em mensurá-lo, causam impactos muito negativos na vida dos indivíduos afetados, além de limitar um maior conhecimento desse sintoma e sua relação com outros fatores como a perda auditiva, por exemplo, dificultando a avaliação dos resultados terapêuticos, reduzindo a concentração e o sono e comprometendo o equilíbrio emocional e a vida social, fazendo com que esses indivíduos apresentem mais queixas ${ }^{(23,25)}$.

Quanto a perda auditiva, percebe-se que os grupos 2 e 4 apresentaram maior pontuação no questionário, mostrando que o THS pode ser útil na identificação de pacientes com problemas auditivos (Figura 2). Entretanto, mesmo os grupos sem perda, pontuaram nessa escala, o que faz pensar que muitos pacientes apresentam dificuldades auditivas em ambientes com ruído. Isso pode acontecer uma vez que a compreensão da fala no silêncio pode se apresentar completamente diferente do que em situações de conversa competitiva, e os testes de fala utilizados na avaliação audiológica podem, dessa forma, não serem eficientes em detectar como se encontra a capacidade funcional do indivíduo de perceber e entender a fala em ambientes ruidosos ${ }^{(26)}$.

O fato supracitado também poderia ser explicado pelas possíveis desordens do processamento auditivo central que os indivíduos poderiam apresentar, visto que, a audiometria é um exame que avalia a sensibilidade auditiva diante de frequências pré-estabelecidas, porém não garante a integridade do processamento auditivo central, principalmente para sons encontrados na rotina diária do indivíduo ${ }^{(27)}$.

Em relação à tolerância ao som, todos os grupos relataram algum grau de intolerância sonora, sendo o grupo 3 (sem perda com zumbido) com maior queixa e o grupo 2 (com perda sem zumbido) com a menor (Figura 3). Entre as possíveis etiologias da hiperacusia estão as condições que envolvem o sistema auditivo periférico, como perda auditiva induzida por ruído, doença de Menière, bem como, doenças e síndromes do sistema nervoso central e doenças hormonais e infecciosas, entretanto limiares auditivos normais não refletem necessariamente uma ausência de dano à cóclea ou ao nervo auditivo ${ }^{(28,29)}$.

É possível afirmar que a associação entre hipersensibilidade ao som e zumbido pode ter sérias implicações na pesquisa e no gerenciamento de ambas as condições, sendo que os dois acometimentos podem ter uma influência significativa nos padrões de atividade auditiva em resposta a sons externos e estão relacionados a limitações como dificuldades para dormir, ouvir e dificuldades de concentração, bem como, problemas sociais, tanto no trabalho, quanto nas relações interpessoais ${ }^{(28,30)}$.

Portanto, diante dos fatos apresentados, considerar a utilização do THS para compreender da melhor forma o paciente frente às diferentes queixas audiológicas pode trazer resultados positivos às futuras intervenções das queixas ou até mesmo no maior engajamento do próprio paciente à terapia, visto que, possibilita compreender qual a queixa que mais o afeta e também facilita na compreensão da necessidade do tratamento.

\section{CONCLUSÃO}

Diante da necessidade de compreender e diferenciar as queixas auditivas das queixas de zumbido, bem como em identificar queixas de tolerância sonora dos pacientes, o THS se mostrou como uma possível ferramenta precisa para auxiliar neste processo, uma vez que, segundo os resultados apresentados, os pacientes que apresentavam perda auditiva, pontuaram mais na categoria audição e aqueles que apresentavam zumbido, pontuaram mais na categoria zumbido, além de ser um questionário rápido e de simples aplicação. Também foi possível notar pela análise dos resultados da tolerância sonora que mesmo indivíduos sem queixas auditivas ou de zumbido, podem apresentar queixas de tolerância. Estes indivíduos podem ser alvo de futuros estudos uma vez que esta população, geralmente desconhece qual profissional procurar quando apresenta estas queixas. Desta forma, o desenvolvimento de estudos futuros seria de grande valia para comprovar e ampliar os conhecimentos a respeito da sensibilidade do THS em diferenciar as queixas auditivas, além de queixas quanto a tolerância aos sons.

\section{REFERÊNCIAS}

1. Lessa AH, Costa MJ, Becker KT, Vaucher AVA. Satisfaction of Hearing Aids Users with hearing loss of severe and deep degree. Int Otorhinolaryngol. 2010;14(3):338-45.

2. Da Silva RGB, De Almeida LP. Próteses auditivas por idosos: aspectos psicossociais, adaptação e qualidade de vida. Interações. 2016;3(17):46374.

3. Sanchez TG, Zonata AI, Bittar RSM, Bento RF. Controvérsias sobre a fisiologia do zumbido. International Arq Fund Otohinolaryngol. 1997;1(1):28 .

4. Sanchez TG, De Medeiros IRT, Levy CPD, Ramalho JRO, Bento RF. Tinnitus in normally hearing patients: clinical aspects and repercussions. Rev Bras Otorrinolaringol. 2005;71(4):427-31. http://dx.doi.org/10.1590/ S0034-72992005000400005. PMid:16446955.

5. Dias A, Cordeiro R, Corrente JE. Incômodo causado pelo zumbido medido pelo Questionário de Gravidade do Zumbido. Rev Saude Publica. 2006;40(4):706-11. http://dx.doi.org/10.1590/S0034-89102006000500022. PMid:17063249.

6. Scheffer AR, Mondelli MFCG. Tinnitus and Hearing Survey: cultural adaptation to Brazilian Portuguese. Braz J Otorhinolaryngol. 2019;87(1):2834. http://dx.doi.org/10.1016/j.bjorl.2019.06.009.

7. Langguth B, Landgrebe M, Schlee W, Schecklmann M, Vielsmeier V, Steffens T, et al. Different patterns of hearing loss among tinnitus patients: a latent class analysis of a large sample. Front Neurol. 2017;8(46):1-8. http://dx.doi.org/10.3389/fneur.2017.00046. PMid:28265258.

8. Schlee W, Shekhawat GS. What does tinnitus have to do with hearing loss? Front Young Minds. 2017;5(2):1-7. http://dx.doi.org/10.3389/ frym.2017.00002. PMid:29353626. 
9. Sultana H, Mumtaz N, Dawood T. Type and Degree of Hearing Loss in Patients with Tinnitus. Int J Rehab Sci. 2018;7(1):24-7.

10. Cash TV. Decreased Sound Tolerance (DST): prevalence, clinical correlates and development os a DST assessment instrument [dissertation]. Richmond: Virginia Commonwealth University; 2015. 181 p.

11. Jastreboff MM, Jastreboff PJ. Decreased sound tolerance and Tinnitus Retraining Therapy (TRT). Aust N Z J Audiol. 2002;24(2):74-84. http:// dx.doi.org/10.1375/audi.24.2.74.31105.

12. Jastreboff PJ, Jastreboff MM. Treatments for decreased sound tolerance (hyperacusis and misophonia). Semin Hear. 2014;35(2):105-20. http:// dx.doi.org/10.1055/s-0034-1372527.

13. Henry JA, Griest S, Zaugg TL, Thielman E, Kaelin C, Galvez G, et al. Tinnitus and hearing survey: a screening tool to differentiate bothersome tinnitus from hearing difficulties. Am J Audiol. 2015;24(1):66-77. http:// dx.doi.org/10.1044/2014_AJA-14-0042. PMid:25551458.

14. Benin L, Teixeira AR, Lessa AH, Rosito LPS, Walbrohel I, Picinini $\mathrm{T}$, et al. Zumbido crônico: estudo em indivíduos com e sem perda auditiva. ConScientiae Saúde. 2016;15(4):657-64. http://dx.doi.org/10.5585/conssaude. v15n4.6907.

15. Henry JA, Zaugg TL, Myers PJ, Schmidt CJ, Griest S, Legro MW, et al. Pilot study to develop telehealth tinnitus management for persons with and without traumatic brain injury. J Rehabil Res Dev. 2012;49(7):1025-42. http://dx.doi.org/10.1682/JRRD.2010.07.0125. PMid:23341277.

16. Scheffer AR, Mondelli MFGC. Tinnitus and Hearing Survey: cultural adaptation to Brazilian Portuguese. Rev Bras Otorrinolaringol. 2019. http:// dx.doi.org/10.1016/j.bjorl.2019.06.009. PMid:31422070.

17. Lopes AC, Munhoz GS, Bozza A. Diagnóstico audiológico. In: Boéchat EM, Menezes PL, Couto CM, Frizzo ACF, Scharlach RC, Anastasio ART. Tratado de audiologia. 2. ed. Rio de Janeiro: Guanabara Koogan; 2015. p. 55-178.

18. Raj-Koziak D, Gos E, Rajchel J, Piłka A, Skarżyński H, Rostkowska $\mathrm{J}$, et al. Tinnitus and hearing survey: a polish study of validity and reliability in a clinical. Audiol Neurotol. 2017;22(4-5):197-204. http:// dx.doi.org/10.1159/000481338. PMid:29130955.

19. Henry JA, Zaugg TL, Myers PM, Kendall CJ. Progressive tinnitus management: Clinical handbook for audiologists. San Diego: Plural; 2010.

20. Shargorodsky J, Curhan GC, Farwell WR. Prevalence and characteristics of tinnitus among US adults. Am J Med. 2010;123(8):711-8. http://dx.doi. org/10.1016/j.amjmed.2010.02.015. PMid:20670725.

21. Tunkel DE, Bauer CA, Sun GH, Rosenfeld RM, Chandrasekhar SS, Cunningham ER Jr, et al. Clinical practice guideline: tinnitus.
Otolaryngol Head Neck Surg. 2014;151(2, Suppl. 2):S1-40. http://dx.doi. org/10.1177/0194599814545325. PMid:25273878.

22. McCormack A, Edmondson-Jones M, Somerset S, Hall D. A systematic review of the reporting of tinnitus prevalence and severity. Hear Res. 2016;2016(337):70-9. http://dx.doi.org/10.1016/j.heares.2016.05.009. PMid:27246985.

23. Mores JT, Bozza A, Magni C, Casali RL, Do Amaral MIR. Perfil clínico e implicações do zumbido em indivíduos com e sem perda auditiva. CoDAS. 2019;31(6):e20180029. http://dx.doi.org/10.1590/2317-1782/20192018029. PMid:31644709.

24. Kehrle HM, Sampaio AL, Granjeiro RC, de Oliveira TS, Oliveira CA. Tinnitus annoyance in normal-hearing individuals: correlation with depression and anxiety. Ann Otol Rhinol Laryngol. 2016;125(3):185-94. http://dx.doi.org/10.1177/0003489415606445. PMid:26424781.

25. Serra LS, Granjeiro RC, Braga SC, Oliveira CA, Sampaio AL. Association between suppression of otoacoustic emissions and annoyance levels in tinnitus patients with normal hearing. Int Tinnitus J. 2015;19(2):52-8. http://dx.doi.org/10.5935/0946-5448.20150009. PMid:27186933.

26. Novelli CL, Carvalho NG, Colella-Santos MF. Hearing in Noise Test, HINT-Brazil, in normal-hearing children. Rev Bras Otorrinolaringol. 2018;84(3):360-7. http://dx.doi.org/10.1016/j.bjorl.2017.04.006. PMid:28549874.

27. Musiek FE, Shinn J, Chermak GD, Bamiou DE. Perspectives on the puretone audiogram. J Am Acad Audiol. 2017;28(7):665-71. PMid:28722648.

28. Fackrell K, Fearnley C, Hoare DJ, Sereda M. Hyperacusis Questionnaire as a tool for measuring hypersensitivity to sound in a tinnitus research population. Biomed Res Int. 2015;2015:1-12.

29. Shim HJ, An Y-H, Kim DH, Yoon JE, Yoon JH. Comparisons of auditory brainstem response and sound level tolerance in tinnitus ears and non-tinnitus ears in unilateral tinnitus patients with normal audiograms. PLoS One. 2017;12(12):e0189157. http://dx.doi.org/10.1371/journal.pone.0189157. PMid:29253030.

30. Aazh H, Lammaing K, Moore BCJ. Factors related to tinnitus and hyperacusis handicap in older people. Int J Audiol. 2017;56(9):1-8. http://dx.doi.org/1 0.1080/14992027.2017.1335887. PMid:28625091.

\section{Contribuição dos autores}

ARS foi responsável pela coleta, análise e interpretação dos dados, análise do conteúdo teórico, e redação do artigo; $M C F$ foi responsável pela redação do artigo, revisão crítica, submissão e trâmites do artigo; MFCGM participou na condição de orientadora, da concepção e projeto do estudo, análise dos dados e correção e aprovação da versão final do artigo. 


\section{ANEXO 1. QUESTIONÁRIO TINNITUS AND HEARING SURVEY BRASILEIRO ${ }^{(16)}$.}

Tinnitus and Hearing Survey Brasileiro

\begin{tabular}{|c|c|c|c|c|c|}
\hline A- Zumbido & $\begin{array}{l}\text { Não, não é um } \\
\text { problema. }\end{array}$ & $\begin{array}{l}\text { Sim, é um problema } \\
\text { pequeno. }\end{array}$ & $\begin{array}{c}\text { Sim, é um problema } \\
\text { moderado. }\end{array}$ & $\begin{array}{c}\text { Sim, é um } \\
\text { problema grande. }\end{array}$ & $\begin{array}{c}\text { Sim, é um problema } \\
\text { muito grande. }\end{array}$ \\
\hline $\begin{array}{l}\text { Na última semana, o zumbido me } \\
\text { impediu de dormir. }\end{array}$ & 0 & 1 & 2 & 3 & 4 \\
\hline $\begin{array}{l}\text { Na última semana, o zumbido } \\
\text { me impediu de me concentrar na } \\
\text { leitura. }\end{array}$ & 0 & 1 & 2 & 3 & 4 \\
\hline $\begin{array}{l}\text { Na última semana, o zumbido me } \\
\text { impediu de relaxar. }\end{array}$ & 0 & 1 & 2 & 3 & 4 \\
\hline $\begin{array}{l}\text { Na última semana, não consegui } \\
\text { tirar o zumbido da minha cabeça. }\end{array}$ & 0 & 1 & 2 & 3 & 4 \\
\hline \multicolumn{6}{|l|}{ TOTAL DE CADA COLUNA } \\
\hline \multicolumn{6}{|l|}{ TOTAL GERAL } \\
\hline B- Audição & $\begin{array}{l}\text { Não, não é um } \\
\text { problema. }\end{array}$ & $\begin{array}{l}\text { Sim, é um problema } \\
\text { pequeno. }\end{array}$ & $\begin{array}{c}\text { Sim, é um problema } \\
\text { moderado. }\end{array}$ & \begin{tabular}{|c|} 
Sim, é um \\
problema grande.
\end{tabular} & $\begin{array}{c}\text { Sim, é um problema } \\
\text { muito grande. }\end{array}$ \\
\hline $\begin{array}{l}\text { Na última semana, eu não } \\
\text { consegui entender o que as } \\
\text { pessoas estavam dizendo em um } \\
\text { lugar barulhento ou com muitas } \\
\text { pessoas. }\end{array}$ & 0 & 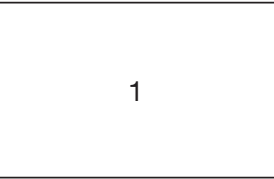 & 2 & 3 & 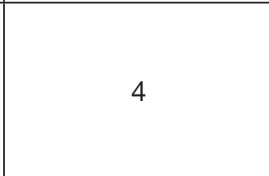 \\
\hline $\begin{array}{c}\text { Na última semana, eu não } \\
\text { consegui entender o que as } \\
\text { pessoas estavam dizendo na TV ou } \\
\text { nos filmes. }\end{array}$ & 0 & 1 & 2 & 3 & 4 \\
\hline $\begin{array}{c}\text { Na última semana eu não entendi } \\
\text { pessoas que falavam baixo. }\end{array}$ & 0 & 1 & 2 & 3 & 4 \\
\hline $\begin{array}{c}\text { Na última semana, eu não } \\
\text { consegui entender o que estava } \\
\text { sendo dito em uma conversa com } \\
\text { várias pessoas juntas (ou conversa } \\
\text { em grupo). }\end{array}$ & 0 & 1 & 2 & 3 & 4 \\
\hline \multicolumn{6}{|l|}{ TOTAL DE CADA COLUNA } \\
\hline \multicolumn{6}{|l|}{ TOTAL GERAL } \\
\hline C- Tolerância ao som & $\begin{array}{l}\text { Não, não é um } \\
\text { problema. }\end{array}$ & $\begin{array}{l}\text { Sim, é um problema } \\
\text { pequeno. }\end{array}$ & $\begin{array}{c}\text { Sim, é um problema } \\
\text { moderado. }\end{array}$ & \begin{tabular}{|c|} 
Sim, é um \\
problema grande.
\end{tabular} & $\begin{array}{c}\text { Sim, é um problema } \\
\text { muito grande. }\end{array}$ \\
\hline $\begin{array}{c}\text { Na última semana, os sons } \\
\text { estavam muito altos ou } \\
\text { desconfortáveis para mim } \\
\text { enquanto eles pareciam } \\
\text { confortáveis para as pessoas em } \\
\text { volta* (Pessoas mastigando, papel } \\
\text { amassando) }\end{array}$ & 0 & 1 & 2 & 3 & 4 \\
\hline \multicolumn{6}{|c|}{ Se você respondeu 1, 2, 3 ou 4 à declaração acima: } \\
\hline \multirow[t]{2}{*}{$\begin{array}{l}{ }^{*} \text { Por favor, liste dois exemplos } \\
\text { de sons que são muito altos ou } \\
\text { desconfortáveis para você, mas } \\
\text { parecem normais para os outros }\end{array}$} & & & & & \\
\hline & \multicolumn{5}{|c|}{$\begin{array}{l}\text { * Se os sons estiverem desconfortáveis durante o uso do aparelho auditivo, favor consultar seu } \\
\text { fonoaudiólogo. }\end{array}$} \\
\hline $\begin{array}{l}\text { Apenas para uso do profissional } \\
\text { (II): }\end{array}$ & & M & $\mathrm{H}$ & & $\mathrm{N}$ \\
\hline
\end{tabular}

